\title{
reviscafuences
}

ISSN: $1575-7072$ | e-ISSN: 2172-7775

Páginas: $15-27$

Recibido: 2021-05-06

Revisado: 2021-08-10

Aceptado: 2021-10-16

Preprint: 2022-01-01

www.revistascientificas.us.es/index.php/fuentes/index

Publicación Final: 2022-01-15

DOI: https://doi.org/10.12795/revistafuentes.2022.16191

\section{Adaptación y validación de un cuestionario para analizar ciberplagio en trabajos académicos universitarios}

\author{
Adaptation and validation of a questionnaire to analyze cyberplagiarism in \\ university academic works
}

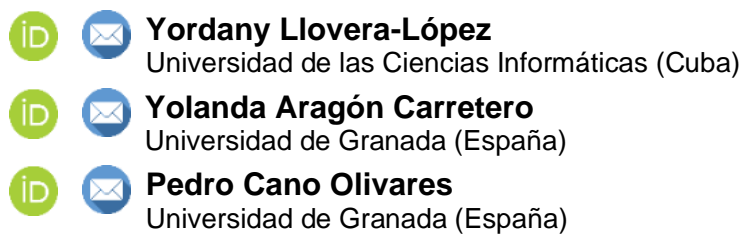

\section{Resumen}

En la era digital el plagio académico ha alcanzado nuevas características y una preocupante dimensión, siendo el ciberplagio su manifestación más agravada. Esta práctica deshonesta se ha enraizado entre el estudiantado universitario a la hora de realizar sus trabajos académicos. Es un fenómeno complejo, con múltiples elementos asociados e interrelacionados, de ahí que las técnicas de investigación han sido variadas, acentuándose el uso de instrumentos auto descriptivos (escalas y cuestionarios). Aunque la mayoría han sido validados, evaluar la calidad de sus propiedades psicométricas en correspondencia con la nueva población de estudio es fundamental para no incurrir en la improvisación. Este artículo describe el proceso de adaptación, validación y fiabilidad de un cuestionario para analizar ciberplagio en trabajos académicos universitarios. Para su desarrollo se siguió la secuencia metodológica de Lacave et al. (2015). Se aplicaron pruebas de validación psicométricas para analizar su fiabilidad y validez, obteniendo resultados adecuados. Además, el Análisis Factorial Exploratorio permitió concretar un cuestionario compuesto por 5 factores y 29 ítems. Su aplicación permitirá analizar la situación del ciberplagio en los trabajos académicos universitarios permitiendo, no solo describir el fenómeno, sino establecer una relación causa-efecto entre los factores. Será un aporte importante para lograr una aproximación al estado de la cuestión en el contexto cubano, donde la problemática ha sido poco explorada.

\section{Abstract}

In the digital age, academic plagiarism has reached new characteristics and a worrying dimension, with cyber-plagiarism being its most aggravated manifestation. This dishonest practice has taken root among university students when carrying out their academic work. It is a complex phenomenon, with multiple associated and interrelated elements, hence the research techniques have been varied, emphasizing the use of self-descriptive instruments (scales and questionnaires). Although most have been validated, evaluating the quality of their psychometric properties in correspondence with the new study population is essential to avoid improvisation. This article describes the process of adaptation, validation and reliability of a questionnaire to analyze cyberplagiarism in university academic papers. For its development, the methodological sequence of Lacave et al. (2015). Psychometric validation tests were applied to analyze their reliability and validity, obtaining adequate results. In addition, the Exploratory Factor Analysis made it possible to specify a questionnaire made up of 5 factors and 29 items. Its application will allow analyzing the situation of cyber plagiarism in university academic works, allowing not only to describe the phenomenon, but also to establish a causal relationship between the factors. It will be an important contribution to achieve an approach to the state of the question in the Cuban context, where the problem has been little explored.

\section{Palabras clave / Keywords}

Fraude Académica, Internet, Trabajos Académicos, Enseñanza Superior. Academic Fraud, Internet, Academic Works, Higher Education. 


\section{Introducción}

En las últimas décadas la Deshonestidad Académica (DA) ha adquirido nuevas características, fundamentalmente los aspectos relativos al plagio académico (Sureda y Comas, 2007b). La cantidad de información proveniente de diferentes fuentes, en su mayoría digital y consultada en Internet, ha llevado al estudiantado a buscar las formas más cómodas para realizar sus trabajos académicos (Rogerson y McCarthy, 2017). Ello ha promovido una práctica académicamente incorrecta definida como ciberplagio (Sureda y Comas, 2007).

En el caso de los campos universitarios, este fenómeno se ha declarado una práctica extendida en los últimos años (Cortés-Vera, 2019). Esto ha suscitado el aumento de su estudio en diferentes regiones geográficas (EE. UU, Reino Unido, Asia, Australia, India, España y Latinoamérica). Se ha catalogado como un problema común y generalizado a todos los niveles (Torres-Diaz et al., 2018), también como grave pues los índices que arrojan las investigaciones son probablemente menores que los existentes en la esfera académica (Rogerson y McCarthy, 2017).

La problemática se ha centrado en las estrategias de búsqueda, selección y manejo de la información en un entorno de gran cantidad de recursos, siendo Internet la principal fuente de información del estudiante (Rogerson y McCarthy, 2017; Torres-Diaz et al., 2018) y el formato electrónico el más utilizado para desarrollar los trabajos (Catro et al., 2019; Espiñeira-Bellón et al., 2021). Sus causas y factores se han clasificado atendiendo a características y comportamientos del profesorado, del estudiantado, de la universidad, a determinados valores sociales predominantes y al desarrollo de las TIC (Awasthi, 2019; Comas y Sureda, 2016; Fatima et al., 2019). Esto ha influenciado las actitudes del estudiante hacia el plagio, percibiéndose una tendencia a subestimar la importancia del fenómeno en los trabajos académcos y sus consecuencias negativas (Belli et al., 2020). De ahí que, las investigaciones se han dirigido a conocer su prevalencia y prácticas frecuentes, así como las percepciones del estudiantado teniendo en cuenta los factores predictivos asociados a este comportamiento (actitudes positivas, actitudes negativas y normas subjetivas).

El conjunto de elementos abordados hasta aquí, describe el plagio como un fenómeno complejo, con múltiples elementos asociados e interrelacionados que lo configuran (Cortés-Vera, 2019). Es un constructo basado en principios ético-morales y, por consiguiente, asociado a una época, una cultura y una sociedad determinada (Kibler, 1993, citado en Comas et al., 2011, p. 208). Por lo que las técnicas de investigación han sido variadas, acentuándose el uso de instrumentos auto descriptivos (escalas y cuestionarios). Los analizados comprenden desde variables más generales como DA (Oran et al., 2016) hasta otras más específicas propias del plagio tradicional y digital (Brennan, 2015; Catro et al., 2019; Mut, 2011; Pupovac et al., 2010; Simeon, 2014). Acentuando el criterio de Husain et al., (2017), en su mayoría han empleado diseños de investigación descriptivos que solo refieren las variables y no establecen una relación causa-efecto entre ellas. Solo uno de ellos evalúa las habilidades informacionales del estudiante para desarrollar un trabajo académico (Mut, 2011), tema al que se le confiere gran importancia para prevenir el plagio en la era digital (Olivia-Dumitrina et al., 2019)

Aunque todos presentan validez y fiabilidad, no son cualidades que se puedan definir en términos absolutos, deben ser comprendidas a partir de los datos obtenidos mediante los análisis estadísticos particulares (Lacave et al., 2015). Es fundamental, tanto para la confección como para la adaptación de un cuestionario, evaluar la calidad de sus propiedades psicométricas en correspondencia con la población de estudio. Esto evitaría incurrir en la improvisación, uno de los factores que puede afectar la fiabilidad y la validez de un instrumento (Hernández-Sampieri et al., 2014).

Partiendo del contexto descrito, se trazó como objetivo describir el proceso de adaptación, validación y fiabilidad de un cuestionario para analizar ciberplagio en trabajos académicos universitarios.

\section{Metodología}

La calidad de un instrumento de medición debe contar con dos requisitos esenciales: validez y fiabilidad (Hernández-Sampieri et al., 2014). Siendo el primero el grado en que un instrumento mide realmente la variable que se busca medir y el segundo el grado en que produce resultados consistentes y coherentes. Nuestro trabajo aborda primero, el proceso de selección y adaptación del cuestionario a partir de la revisión de literatura. Seguidamente, evalúa su calidad a partir del análisis de validez y fiabilidad siguiendo la secuencia metodológica de (Lacave et al., 2015) (Figura 1). 


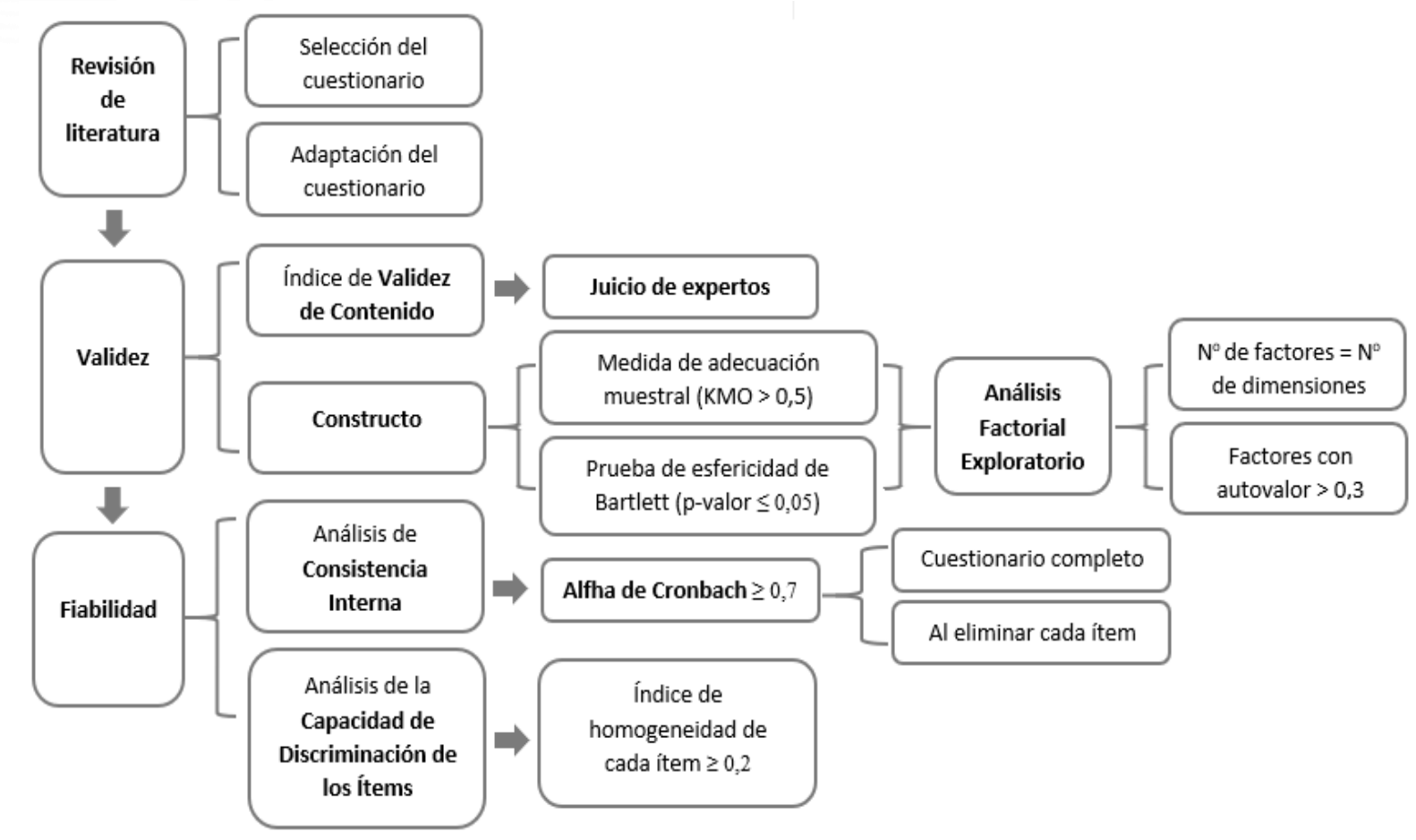

Figura 1. Secuencia metodológica. Adaptado de Lacave et al. (2015)

\subsection{Participantes}

Por un lado, se trabajó con 12 expertos en la técnica Delphi, provenientes de 5 universidades ubicadas en España, Costa Rica, México y Cuba. Se consideraron parámetros metodológicos comunes en esta técnica: el número de expertos, la disposición para participar y la calidad del panel (López-Gómez, 2018; MengualAndrés et al., 2016). Aunque existe diversidad de criterios sobre la cantidad de expertos pertinentes, para algunos autores entre 10 y 15 es aceptable (Landeta, 1999). De ellos, 7 con formación de grado académico de doctor y 5 de máster. Su experiencia profesional se sustenta en la investigación y la docencia, además, cuentan con publicaciones relacionadas con la temática. Su especialidad se centra en las siguientes áreas: Investigación Didáctica (3), Pedagogía Aplicada y Psicología de la Educación (2), Derecho (1), Filología (1), Tecnología Educativa (3) y Ciencias de la Información (2). La antigüedad laboral de 8 expertos oscila entre 16 y 30 años y 4 entre 15 y 20 años de experiencia. La calidad del panel fue constatada a través del índice de Competencia Experta (K), para lo que se siguió el procedimiento de Cabero-Almenara y Barroso-Osuna (2013).

Por otra parte, se aplicó un estudio piloto. La muestra, no probabilística e intencional, estuvo conformada por 200 estudiantes, $63,5 \%$ hombres y $36,5 \%$ mujeres, matriculados en segundo $(27,5 \%)$, tercer $(30 \%)$, cuarto (24\%) y quinto curso $(18,5 \%)$ de la carrera Ingeniería en Ciencias Informáticas de la Universidad de las Ciencias Informáticas (Cuba). El tamaño de la muestra es considerado aceptable para el análisis descriptivo y psicométrico de los ítems (Lloret-Segura et al., 2014). No se destinó al primer curso teniendo en cuenta que uno de los requisitos era haber culminado al menos un curso académico. De esta manera tendrían mayor tiempo de estancia en la institución y experiencia en la elaboración de trabajos académicos para responder las preguntas.

\subsection{Instrumento}

Se seleccionó el cuestionario de Mut (2011) orientado a analizar el ciberplagio en el desarrollo de trabajos académicos. Fue adaptado teniendo en cuenta las características del contexto de aplicación. Se prestó atención al vincular los términos entre la cultura de origen y la cultura destinataria, evitando factores que pudieran afectar su fiabilidad y validez (Hernández-Sampieri et al., 2014). Se incluyeron 4 ítems del cuestionario de actitud hacia el plagio de Pupovac et al. (2010) y 7 ítems de elaboración propia a partir de la 
revisión bibliográfica (Tabla 1). Aunque está dirigido a enseñanza media, ha sido seleccionado por las razones siguientes:

- El diseño e implementación metodológica de la investigación de Mut (2011) se ajusta al propósito de nuestro estudio.

- Permite una aproximación al estado de la cuestión a partir de cuatro dimensiones de estudio, estableciendo una relación entre ellas.

- La calidad del cuestionario se constató mediante la validez interna (abarca todas las posibilidades explicativas de la investigación), externa (utilización de muestreo aleatorio y casos modales, lo que permitió obtener un cuestionario aplicable a otros contextos) y de constructo (profunda revisión de literatura y fundamentación teórica).

- Incluye indicadores que permiten obtener información relativa al nivel de desarrollo de la competencia informacional para hacer un uso eficaz y ético de la información.

- Los resultados reflejan una realidad sobre la existencia de prácticas de ciberplagio y causas asociadas, que no difiere notablemente de los obtenidos en estudios universitarios (Cebrián-Robles et al., 2018; Comas \& Sureda, 2010; Singh, 2017).

Tabla 1

Ítems incluidos en el cuestionario de Mut (2011)

\begin{tabular}{|c|c|c|}
\hline ITEMS & & FUENTE \\
\hline $\begin{array}{l}- \\
- \\
- \\
- \\
-\end{array}$ & $\begin{array}{l}\text { A veces tengo la tentación de plagiar, pues los demás lo están haciendo. } \\
\text { Sigo plagiando porque todavía no he sido atrapado. } \\
\text { En tiempos de declive moral y ético, es importante debatir temas como el plagio } \\
\text { y el ciberplagio entre el estudiantado. } \\
\text { El plagio empobrece el espíritu investigador. } \\
\text { Soy consciente de la implicación de cometer ciberplagio. } \\
\text { Los trabajos académicos (manuales, ejercicios de examen o trabajos, informes, } \\
\text { artículos científicos, proyectos, etc.) son una herramienta importante para } \\
\text { desarrollar el aprendizaje. } \\
\text { La universidad ha ofertado cursos para desarrollar conocimientos y habilidades } \\
\text { para hacer uso de la información de forma ética y legal. } \\
\text { Los profesores comprueban los trabajos académicos utilizando un software } \\
\text { antiplagio, para detectar citas incorrectas o posible plagio. } \\
\text { Siempre consulto los estilos y normas de redacción de bibliografías, para elaborar } \\
\text { correctamente las citas y referencias bibliográficas en un trabajo académico. } \\
\text { Conozco el protocolo de las citas textuales y sus diferencias con las citas } \\
\text { parafraseadas. } \\
\text { Conozco las normas establecidas para hacer un uso ético y legal de la } \\
\text { información, por ejemplo (el Derecho de Autor y las Licencias Creative } \\
\text { Commons). }\end{array}$ & $\begin{array}{l}\text { Elaboración propia } \\
\text { Elaboración propia } \\
\text { Elaboración propia } \\
\text { Elaboración propia } \\
\text { Elaboración propia }\end{array}$ \\
\hline
\end{tabular}

Se conformó un cuestionario mixto compuesto por tres bloques, dos de ellos subdivididos siguiendo un criterio de agrupación por dimensiones. El Bloque I consiste en 5 preguntas introductorias de carácter sociodemográfico y tres orientadas a conocer: situación académica del estudiante en el presente curso, autopercepción sobre sus habilidades en el manejo de las TIC y conocimiento del reglamento docente disciplinario de la institución.

El Bloque II está compuesto por preguntas estructuradas de alternativa múltiple para conocer la prevalencia o no de ciberplagio académico. Se divide en dos partes, una está referida al comportamiento propio del estudiante y la otra al comportamiento atribuido a sus compañeros. El rango de frecuencia de respuesta oscila entre: a) nunca, b) entre una y dos veces, c) entre tres y cinco veces, d) entre seis y diez veces y e) más de diez veces.

El Boque III es de preguntas en formato escala psicométrica Likert de cinco puntos, donde 1 equivale a "totalmente en desacuerdo" y 5 a " totalmente de acuerdo". Están orientadas a conocer las causas asociadas a la comisión de ciberplagio académico, la actitud hacia el ciberplagio académico y el desarrollo de habilidades informacionales. 


\section{Análisis de validez y fiabilidad}

\subsection{Validez de contenido y de constructo}

El método Delphy se ajustó a las etapas de Blasco y López (2010): Preliminar, Exploratoria y Final (Figura 2).

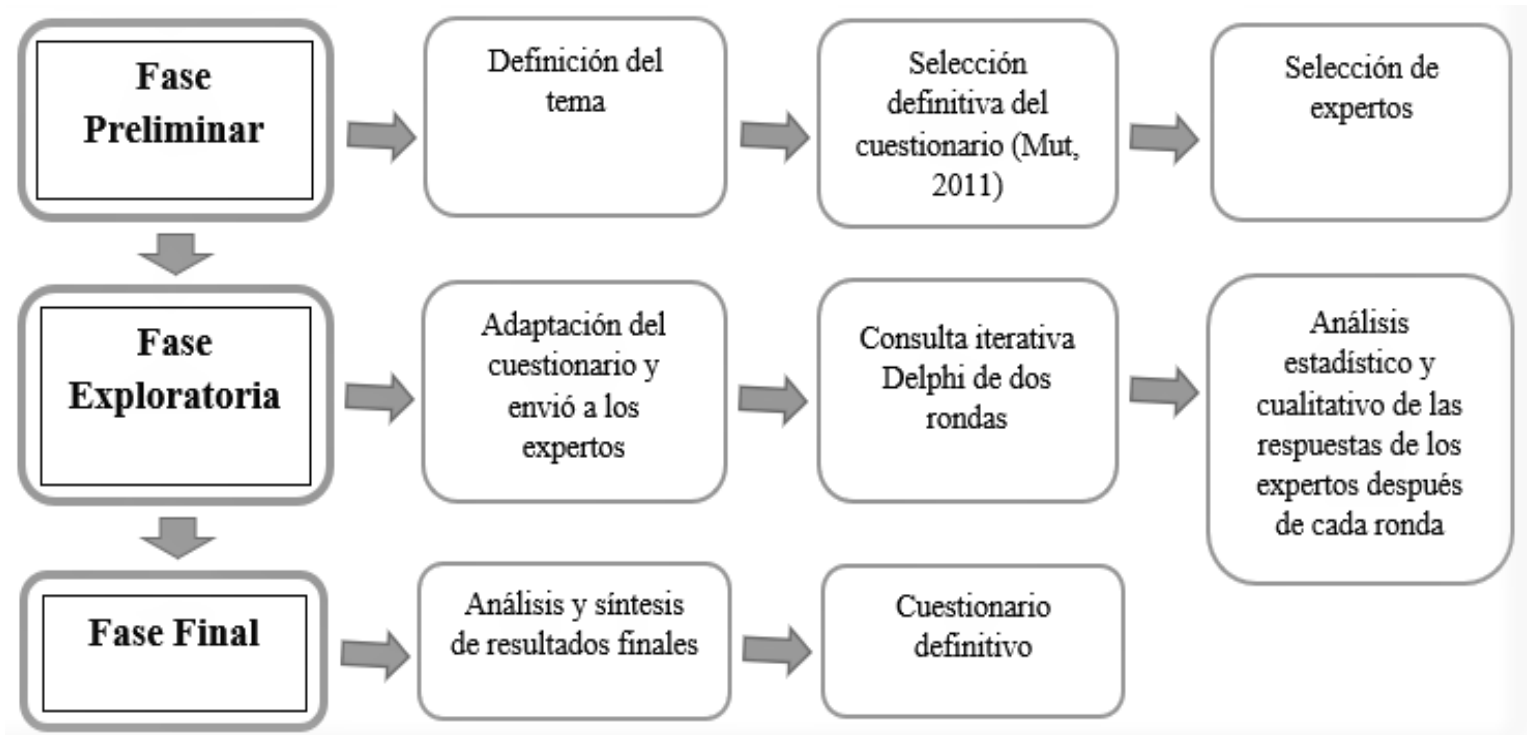

Figura 2. Fases del Delphy. Elaboracion propia

Siguiendo las recomendaciones de Keeney et al., (2006), se realizó una consulta iterativa Delphi de dos rondas con expertos para constatar que los ítems sean una muestra representativa del contenido a evaluar (Prieto y Delgado, 2010). Solo se muestran los resultados de los Boques II y III, puesto que el Bloque I indaga fundamentalmente aspectos sociodemográficos. El análisis de los datos en ambas rondas (R1 y R2) demostró un amplio acuerdo sobre los ítems evaluados. Con el objetivo de realizar correcciones a partir de las valoraciones cualitativas de mayor consenso, se llevó a cabo el análisis de la R1. Resumimos algunas de ellas:

1. El ítem 1 de la dimensión 1 no está orientado la ciberplagio, sino al fraude tradicional.

2. El ítem 4 de la dimensión 2 no procede, se considera que puede obtenerse la información con el ítem 3.

3. El ítem 9 de la dimensión 2 en principio no se considera un motivo de plagio, en todo caso una falta de acierto del docente en los trabajos orientados.

4. Sugieren añadir a la redacción de algunos ítems las siguientes coletillas:

5. Ítem 6 de la dimensión 1 "o sin aportar permisos de reproducción o adaptación".

6. Ítem 6 de la dimensión 2 "sin ninguna guía ".

7. Ítem 14 de la dimensión 4 "Derecho de Autor y las Licencias Creative Commons".

8. Ítem 15 " de la dimensión 4 "APA o cualquier otro sistema", pues existen varios estilos y normas de redacción de bibliografías.

Para orientar el análisis de datos y la toma de decisiones se establecieron dos criterios: la medida del consenso y de la estabilidad en las respuestas del panel (López-Gómez, 2018). Durante el análisis cuantitativo se siguió la metodología de Mengual-Andrés et al. (2016): a) Tendencia central y medidas de dispersión; b) Análisis del nivel de consenso entre rondas (IQR) y c) Análisis de la estabilidad del grupo en las respuestas (Prueba de los rangos con signo de Wilcoxon). Para seleccionar los ítems definitivos se tuvieron en cuenta los criterios de consenso establecidos por los autores anteriores y se adaptaron los valores para una escala Likert de 4 puntos (Tabla 2).

El análisis de los resultados de las dos rondas Delphi se presentan a través de una tabla de contingencia (Tabla 3). Los ítems que respondieron a un amplio grado de consenso se han identificado con una $A$ y los 
descartados con una D. En la dimensión 1 fue descartado 1 ítem quedando 7 de los 8 propuestos inicialmente. En la dimensión 2 fueron descartados 3 quedando 11 de los 14 propuestos inicialmente. La estructura de las dimensiones 3 y 4 no sufrió modificaciones.

\section{Tabla 2}

Criterios para la definición de consenso. Panel Delphi

CONSENSO PARÁMETROS (Mdn: Mediana; IQR: Rango Intercuartil y Frecuencia)

\begin{tabular}{ll}
\hline Acuerdo (A) & $M d n \geq 2.5, I Q R \leq 1.5$ \\
& $M d n \geq 2.5, I Q R \leq 2$, frecuencia $(3-4) \geq 70 \%$ \\
& $M d n \leq 2, I Q R \leq 1.5$ \\
Desacuerdo (D) & $M d n \leq 2, I Q R \leq 2$, frecuencia $(1-2) \geq 70 \%$ \\
& $M d n \geq 2, I Q R \leq 2$ \\
\hline Neutral $(N)$ & $M$ \\
\hline
\end{tabular}

Nota: Adaptada de Mengual-Andrés, S., Roig-Vila, R. y Blasco, J. (2016). Criterios de consenso establecidos para selección definitiva de los ítems.

\section{Tabla 3}

Tabla de contingencia para el análisis de los resultados obtenidos durante las rondas Delphi

\begin{tabular}{|c|c|c|c|c|c|c|c|c|c|}
\hline & R1 & & & & $\mathbf{R 2}$ & & & & Consenso \\
\hline & $\mathbf{M}$ & Me & SD & IQR & $\mathbf{M}$ & Me & SD & IQR & \\
\hline \multicolumn{10}{|c|}{ Dimensión 1} \\
\hline$\overline{\mathrm{It} 1}$ & 1.86 & 2.00 & .690 & 1.00 & 1.29 & 1.00 & .488 & 1.00 & $\mathrm{D}$ \\
\hline It2 & 3.71 & 4.00 & .488 & 1.00 & 3.71 & 4.00 & .488 & 1.00 & $A$ \\
\hline It3 & 4.00 & 4.00 & 0.000 & 0.00 & 4.00 & 4.00 & 0.000 & 0.00 & $A$ \\
\hline It4 & 4.00 & 4.00 & 0.000 & 0.00 & 4.00 & 4.00 & 0.000 & 0.00 & $A$ \\
\hline It5 & 4.00 & 4.00 & 0.000 & 0.00 & 4.00 & 4.00 & 0.000 & 0.00 & $A$ \\
\hline It6 & 3.71 & 4.00 & .488 & 1.00 & 3.86 & 4.00 & .3780 & 0.00 & $A$ \\
\hline It7 & 4.00 & 4.00 & 0.000 & 0.00 & 4.00 & 4.00 & 0.000 & 0.00 & $A$ \\
\hline It8 & 3.86 & 4.00 & .378 & 0.00 & 4.00 & 4.00 & 0.000 & 0.00 & $A$ \\
\hline \multicolumn{10}{|c|}{ Dimensión 2} \\
\hline It1 & 3.29 & 3.00 & .756 & 1.00 & 3.57 & 4.00 & .535 & 1.00 & $A$ \\
\hline It2 & 3.14 & 3.00 & .690 & 1.00 & 3.14 & 3.00 & .690 & 1.00 & $A$ \\
\hline It3 & 3.71 & 4.00 & .488 & 1.00 & 3.57 & 4.00 & .535 & 1.00 & $A$ \\
\hline It5 & 3.71 & 4.00 & .488 & 1.00 & 3.71 & 4.00 & .488 & 1.00 & $A$ \\
\hline It6 & 3.71 & 4.00 & .488 & 1.00 & 3.71 & 4.00 & .488 & 1.00 & $A$ \\
\hline It7 & 3.71 & 4.00 & .488 & 1.00 & 3.86 & 4.00 & .378 & 0.00 & $A$ \\
\hline It8 & 3.71 & 4.00 & .488 & 1.00 & 3.86 & 4.00 & .378 & 0.00 & $A$ \\
\hline
\end{tabular}




\begin{tabular}{|c|c|c|c|c|c|c|c|c|c|}
\hline It9 & 1.86 & 2.00 & .690 & 1.00 & 1.71 & 2.00 & .756 & 1.00 & D \\
\hline It10 & 3.43 & 3.00 & .535 & 1.00 & 3.71 & 4.00 & .488 & 1.00 & A \\
\hline It11 & 2.86 & 3.00 & .900 & 1.00 & 3.14 & 3.00 & .378 & 0.00 & A \\
\hline It12 & 2.43 & 2.00 & .535 & 1.00 & 2.29 & 2.00 & .488 & 1.00 & D \\
\hline It13 & 4.00 & 4.00 & 0.000 & 0.00 & 4.00 & 4.00 & 0.000 & 0.00 & A \\
\hline It14 & 4.00 & 4.00 & 0.000 & 0.00 & 4.00 & 4.00 & 0.000 & 0.00 & A \\
\hline \multicolumn{10}{|c|}{ Dimensión 3} \\
\hline It1 & 4.00 & 4.00 & 0.000 & 0.00 & 4.00 & 4.00 & 0.000 & 0.00 & $A$ \\
\hline It2 & 3.86 & 4.00 & .378 & 0.00 & 4.00 & 4.00 & 0.000 & 0.00 & A \\
\hline It3 & 2.29 & 2.00 & .756 & 1.00 & 3.71 & 4.00 & .488 & 1.00 & A2 \\
\hline It4 & 4.00 & 4.00 & 0.000 & 0.00 & 4.00 & 4.00 & 0.000 & 0.00 & A \\
\hline It5 & 4.00 & 4.00 & 0.000 & 0.00 & 4.00 & 4.00 & 0.000 & 0.00 & A \\
\hline It6 & 2.00 & 2.00 & .816 & 2.00 & 3.86 & 4.00 & .378 & 0.00 & A2 \\
\hline It7 & 4.00 & 4.00 & 0.000 & 0.00 & 4.00 & 4.00 & 0.000 & 0.00 & A \\
\hline It8 & 4.00 & 4.00 & 0.000 & 0.00 & 4.00 & 4.00 & 0.000 & 0.00 & A \\
\hline It9 & 4.00 & 4.00 & 0.000 & 0.00 & 4.00 & 4.00 & 0.000 & 0.00 & A \\
\hline \multicolumn{10}{|c|}{ Dimensión 4} \\
\hline It1 & 3.14 & 3.00 & .378 & 0.00 & 3.29 & 3.00 & .488 & 1.00 & A \\
\hline It2 & 3.86 & 4.00 & .378 & 0.00 & 3.86 & 4.00 & .378 & 0.00 & A \\
\hline It3 & 1.86 & 2.00 & .378 & 0.00 & 1.71 & 2.00 & .488 & 1.00 & A \\
\hline It4 & 3.86 & 4.00 & .378 & 0.00 & 4.00 & 4.00 & 0.000 & 0.00 & A \\
\hline It5 & 3.71 & 4.00 & .488 & 1.00 & 3.86 & 4.00 & .378 & 0.00 & A \\
\hline It7 & 3.57 & 4.00 & .535 & 1.00 & 3.43 & 3.00 & .535 & 1.00 & A \\
\hline It8 & 3.71 & 4.00 & .488 & 1.00 & 3.86 & 4.00 & .378 & 0.00 & A \\
\hline It9 & 3.71 & 4.00 & .488 & 1.00 & 3.71 & 4.00 & .488 & 1.00 & A \\
\hline It10 & 3.14 & 3.00 & .378 & 0.00 & 3.29 & 3.00 & .488 & 1.00 & A \\
\hline It11 & 3.29 & 3.00 & .488 & 1.00 & 3.43 & 3.00 & .535 & 1.00 & A \\
\hline It12 & 4.00 & 4.00 & 0.000 & 0.00 & 4.00 & 4.00 & 0.000 & 0.00 & A \\
\hline It13 & 4.00 & 4.00 & 0.000 & 0.00 & 4.00 & 4.00 & 0.000 & 0.00 & A \\
\hline It14 & 3.86 & 4.00 & .378 & 0.00 & 4.00 & 4.00 & 0.000 & 0.00 & A \\
\hline It15 & 3.57 & 4.00 & .535 & 1.00 & 3.71 & 4.00 & .488 & 1.00 & A \\
\hline It16 & 3.71 & 4.00 & .488 & 1.00 & 3.71 & 4.00 & .488 & 1.00 & A \\
\hline It17 & 3.71 & 4.00 & .488 & 1.00 & 3.86 & 4.00 & .378 & 1.00 & A \\
\hline
\end{tabular}




$\begin{array}{lllllllllll}\text { It18 } & 3.57 & 4.00 & .535 & 1.00 & 3.86 & 4.00 & .378 & 0.00 & \text { A } \\ \text { It19 } & 2.29 & 2.00 & .756 & 1.00 & 3.71 & 4.00 & .488 & 1.00 & \text { A2 } \\ \text { It20 } & 3.71 & 4.00 & .488 & 1.00 & 3.71 & 4.00 & .488 & 1.00 & \text { A }\end{array}$

Nota: Adaptada de Mengual-Andrés, S., Roig-Vila, R. y Blasco, J. (2016). M: Media; SD: Desviación Estándar; Me: Mediana; IQR: Rango Intercuartil Relativo; A: Aceptado en R1 y R2; A2: Neutral en R1 y Aceptado en R2; D: Descartado.

Para comprobar la estabilidad del panel de expertos se aplicó la prueba no paramétrica de rangos con signos de Wilcoxon para muestra relacionadas, pues los datos analizados no cumplen con el supuesto de normalidad. El análisis de la evaluación de los ítems entre rondas, asumiendo un margen de error de 0.05 , confirmó que la mayoría obtuvo un valor $\mathrm{p}>0.05$. Lo anterior permite concluir que se rechaza la $\mathrm{H} 1 \mathrm{pues}$ el criterio de los expertos no produjo cambios significativos en los ítems después de la R2.

Teniendo en cuenta el nivel aceptable de convergencia entre el criterio de los expertos, el tiempo limitado, la demora en la comunicación y la posibilidad de abandono, se determinó la no rentabilidad de una tercera ronda. Esto se corresponde con la revisión de investigaciones educativas de López-Gómez (2018) donde, la mayoría han desarrollado el método Delphi en dos rondas, usualmente en tres y rara vez en más. Tras los análisis la versión adaptada del cuestionario quedó compuesta por 48 ítems, distribuidos en 4 dimensiones (Tabla 4).

\section{Tabla 4}

Dimensiones e indicadores del cuestionario

\begin{tabular}{ll}
\hline DIMENSIONES & INDICADORES \\
\hline Prevalencia de Ciberplagio Académico & Prevalencia declarada (comportamiento del propio estudiante) \\
& Prevalencia atribuida (comportamiento atribuido a otro estudiante) \\
\hline Causas de ciberplagio académico & Causas atribuidas al alumnado (factores internos) \\
& Causas atribuidas al profesorado (factores contextuales) \\
& Causas atribuidas a la institución (factores contextuales) \\
& Causas externas a la práctica educativa (factores externos) \\
\hline Actitud ante el ciberplagio académico & Actitud positiva \\
& Actitud negativa \\
\hline Habilidades informacionales relacionadas con & Conocimientos y habilidades en la elaboración de \\
el desarrollo de trabajos académicos & trabajos académicos \\
& Estrategias de búsqueda documental \\
& Fuentes de documentación utilizadas \\
& Evaluación de la información \\
& Conocimientos de citación \\
\hline
\end{tabular}

Nota: Elaboración con base en Mut (2011)

Posteriormente, se previó realizar el Análisis Factorial Exploratorio (AFE) con el propósito de: primero, comprobar la correcta agrupación de los ítems en las 4 dimensiones definidas inicialmente; segundo, si las dimensiones son suficientes para explicar los resultados que proporcionan las 48 preguntas incluidas; y tercero, realizar los ajustes de factores e ítems necesarios intentando mantener el poder informativo deseado. Para comprobar la viabilidad de esta prueba, se procede al cálculo de la Medida de Adecuación Muestral $(\mathrm{KMO})$, obteniendo un valor de $0,740(>0,5)$ y un valor de 0 en la prueba de esfericidad de Bartlett. El AFE se realizó fijando 4 factores de extracción, en correspondencia con el número de dimensiones del cuestionario inicial. Al analizar la varianza total explicada, los 4 primeros factores son mayores que 1, pero explican solo el $44,488 \%$ de los datos originales. Además, se muestran 3 factores más con valores mayores a 1 que deberían ser extraídos (Tabla 5). En busca de una mejor solución se determinó calcular primero el Alpha de Cronbach para comprobar la fiabilidad del cuestionario. Este análisis podría sugerir un ajuste de ítems, y, por consiguiente, de factores. 
Tabla 5

Autovalores iniciales obtenidos mediante el método de extracción de máxima verosimilitud

\begin{tabular}{ccccccc}
\hline & \multicolumn{3}{c}{ Autovalores iniciales } & \multicolumn{3}{c}{ Sumas de rotación de cargas al cuadrado } \\
Componente & Total & \% de varianza & $\%$ acumulado & Total & \% de varianza & \% acumulado \\
\hline 1 & 5,089 & 21,205 & 21,205 & 3,339 & 13,913 & 13,913 \\
2 & 3,090 & 12,875 & 34,080 & 2,881 & 12,005 & 25,918 \\
3 & 1,922 & 8,009 & 42,089 & 2,345 & 9,772 & 35,690 \\
4 & 1,839 & 7,661 & 49,749 & 2,112 & 8,799 & 44,488 \\
5 & 1,397 & 5,821 & 55,571 & 1,725 & 7,186 & 51,674 \\
6 & 1,228 & 5,118 & 60,689 & 1,559 & 6,495 & 58,169 \\
7 & 1,118 & 4,658 & 65,347 & 1,557 & 6,486 & 64,655 \\
\hline
\end{tabular}

\subsection{Análisis de fiabilidad. Cálculo del Alpha de Cronbach}

El coeficiente Alpha de Cronbach está basado en la correlación inter elementos promedio y asume que los ítems miden un mismo constructo y están correlacionados (Prieto y Delgado, 2010). El índice de fiabilidad del cuestionario completo es de 0,723 , considerado aceptable, pero no el nivel deseado, por lo que se asumieron dos criterios para la eliminación de los ítems. Primero, los ítems con un Alfha de Cronbach $\geq 0,729(10,18$, $23,33,37$ y 48), aumentando este valor a 0,773. Segundo, los ítems con índice de homogeneidad < 0,2 (17, $20,24,25,27,30,35,43,44$ y 45 ), al tener un bajo índice no están midiendo lo mismo que la prueba globalmente (Lacave et al., 2015). Al aplicar estos dos criterios el cuestionario alcanzó un Alfha de Cronbach de 0,851 .

\subsection{Análisis Factorial Exploratorio del cuestionario reducido}

Con la reducción de los ítems mediante el Alfha de Cronbach, el valor KMO pasó a ser de $0,775(>0,5)$ y el valor del nivel de significación de la prueba de esfericidad de Bartlett es 0. Posteriormente, se desarrolló el AFE para identificar el número y composición de los factores comunes (variables latentes) necesarios para explicar la varianza común del conjunto de ítems analizado (Lloret-Segura et al., 2014). Se utilizó el método de extracción (análisis de componentes principales) y cómo método de rotación (Varimax con normalización Kaiser). En un primer momento la rotación convergió en 7 iteraciones. Para encontrar un mejor ajuste de ítems por factores se eliminaron los ítems 3, 6, 28 y 31. Definitivamente se obtuvieron cinco factores que explican el $62,039 \%$ de la varianza total, uno más que los propuestos inicialmente. En busca del modelo que mejor se ajustara a la población de estudio, se asumieron los autovalores mayores a 0,3, una regla general aceptable en la saturación mínima a considerar (Lloret-Segura et al., 2014). En la Tabla 6 se presenta la distribución de los ítems por cada en cada factor antes y después de la última rotación.

\section{Tabla 6}

Estructura factorial exploratoria antes y después de la última rotación

\begin{tabular}{|c|c|c|c|c|c|c|c|c|c|c|}
\hline \multicolumn{6}{|c|}{ Antes de la rotación } & \multicolumn{5}{|c|}{ Después de la rotación } \\
\hline Ítems & 1 & 2 & 3 & 4 & 5 & 1 & 2 & 3 & 4 & 5 \\
\hline It1 &, 511 & & & & & & & ,718 & & \\
\hline It2 & & ,525 & & & & & & 678 & & \\
\hline It4 & & ,546 & & & & & & ,765 & & \\
\hline It5 & ,402 & & & & & & & ,517 & & \\
\hline It7 & & ,447 & & & & & & ,596 & & \\
\hline It8 & 316 & & & & & & ,452 & & & \\
\hline It9 & 842 & & & & & & ,962 & & & \\
\hline It11 & ,464 & & & & & & ,372 & & & \\
\hline It12 & ,364 & & & & & & 498 & & & \\
\hline It13 & & &,- 722 & & & & 949 & & & \\
\hline It14 & & & -,720 & & & & ,949 & & & \\
\hline It15 & & &,- 709 & & & & 945 & & & \\
\hline It16 & 341 & & & & & & ,437 & & & \\
\hline It19 & & ,621 & & & & & ,526 & & & \\
\hline It21 & & & & ,50 & & & & & & ,962 \\
\hline
\end{tabular}




\begin{tabular}{|c|c|c|c|c|c|}
\hline It22 & & 381, & & & ,487 \\
\hline It26 & & 787, & & & ,965, \\
\hline $\begin{array}{l}1+27 \\
\text { It28 }\end{array}$ & , 402 & ,381 & & 387 & \\
\hline It29 & & 618, & & ,674 & \\
\hline It32 & & 787, & & ,965 & \\
\hline It34 & 838, & & 971 & & \\
\hline It36 & 833 & & 978 & & \\
\hline It38 & 847, & & 970, & & \\
\hline It39 & & 465, & & 610, & \\
\hline It 40 & & ,504 & 607, & & \\
\hline It 41 & & ,465 & & 610, & \\
\hline It 46 & & ,381 & & ,387 & \\
\hline It 47 & 840 & & 980, & & \\
\hline
\end{tabular}

Al concluir el AFE y teniendo en cuenta que se eliminaron 4 ítems durante la rotación, se calculó nuevamente el Alfha de Cronbach, aumentando su valor a 0,859. Lo cual indica que el cuestionario definitivo, compuesto por 29 Ítems, tiene buena consistencia interna ya que, cuando el coeficiente es igual o superior a 0.7 es considerado aceptable (Prieto y Delgado, 2010).

\section{Discusión}

Posterior a la revisión de literatura, se seleccionó y adoptó el cuestionario de Mut (2011) para analizar la situación del ciberplagio académico, término que se maneja actualmente para referirse a esta práctica deshonesta (Comas y Sureda, 2016). La evaluación de calidad del instrumento se realizó a través del análisis de validez y fiabilidad, siguiendo la secuencia metodológica de Lacave et al. (2015). La primera adaptación del cuestionario constó de 4 dimensiones y 51 ítems. Luego de su evaluación por los expertos en cuanto a contenido, el número de ítems se redujo a 48 distribuidos en 4 dimensiones: prácticas de ciberplagio, causas de ciberplagio, actitudes hacia el ciberplagio y habilidades informacionales). Tras el cálculo final del Alfha de Cronbach, alcanzó un índice de fiabilidad de 0,859 al eliminar 19 ítems de los 48 propuestos, incluidos aquí los que se eliminaron duranta la rotación en el AFE. De ellos, 15 corresponden al cuestionario de Mut (2011), 2 a los incorporados de Pupovac et al. (2010) y 2 a los de elaboración propia. Esto demuestra que las respuestas dadas por los estudiantes a estas preguntas no aportan información valiosa para medir la magnitud y características de la problemática en cuestión.

El AFE permitió concretar 5 fatores y 29 ítems (Tabla 7). Los ajustes correspondientes al nuevo número de factores mantuvieron los 3 primeros, quedando fragmentado en dos el número 4 relacionado con las habilidades informacionales. El factor 1 mantuvo 5 ítems que se corresponden con las prácticas de ciberplagio más frecuentes: copiar y pegar fragmentos de textos e incluirlos en trabajos propios sin citar la fuente; la citación incorrecta; la descarga y uso desmedido de recursos de Internet asumiéndolos como propios de manera parcial o total (Awasthi, 2019; González-Torres et al., 2018). Con ello, se obtiene información sobre la prevalencia autoreferida y atribuida a los compañeros, siguiendo la misma línea de estudios presdentes (Muñoz-Cantero et al., 2019; Mut, 2011). El factor 2 mantuvo 9 ítems que responden a las causas de ciberplagio, enmarcados dentro de la clasificación de factores predictivos de Comas y Sureda (2010): individuales, contextuales y externos a la práctica educativa. Corroborados recientemente por diferentes estudios (Awasthi, 2019; Boillos, 2020; Catro et al., 2019; Muñoz-Cantero et al., 2019). El factor 3 mantuvo 4 ítems que destacan actitudes positivas y negativas hacia el ciberplagio, considerado hoy un tema central por la influencia significativa que puede ejercer la actitud en los actos de DA (Belli et al., 2020; Husain et al., 2017). El reordenamiento de los ítems en los factores 4 y 5 cobra sentido pues la rotación agrupó, por un lado, los orientados a estrategias y fuentes de búsqueda documental, y, por otro, los correspondientes a conocimientos y habilidades en el manejo de información. En tal sentido, el trabajo de Muñoz-Cantero et al., (2019) aborda solo la citación, por lo que, estos dos factores constituyen una fortaleza de nuestro trabajo. La importancia de indagar en estas cuestiones se refleja en posiciones teóricas que sostienen que, las prácticas plagiarias manifiestan un déficit de competencias para acceder y usar la información de forma eficaz y ética (Comas y Sureda, 2016). Además, el factor formativo es un patrón repetido como causa de plagio en estudios actuales (Cebrián-Robles et al., 2020; Fazilatfar et al., 2018).

Finalmente, el cuestionario para analizar el ciberplagio en los trabajos académicos universitario ofrece mejores propiedades psicométricas, tanto general como por dimensiones, que el cuestionario inicial. 
Evidenciando que, en análisis estadísticos posteriores, los datos proporcionados por las 29 preguntas distribuidas en 5 factores tienen más validez y fiabilidad que los que ofrecen las 48 preguntas distribuidas en los 4 factores iniciales.

\section{Tabla 7}

Dimensiones (factores) e ítems definitivos

Prácticas de ciberplagio académico

It1. Copiar de páginas web fragmentos de texto y sin citar, pegarlos directamente en un documento en el cual hay una parte escrita por ti, y entregarlo como trabajo de una asignatura.

It2. Elaborar íntegramente un trabajo a partir de fragmentos copiados literalmente de páginas Web y de recursos localizados en Internet.

It4. Copiar fragmentos de fuentes impresas (libros, enciclopedias, periódicos, artículos de revista, etc.) y sin citar la fuente, añadirlo como partes de un trabajo propio.

It5. Bajar imágenes de Internet y utilizarlas para un trabajo sin citar la fuente de obtención, o sin aportar permisos de reproducción o adaptación.

It7. Copiar de Internet fragmentos de un trabajo en otro idioma, traducirlos, pegarlos sin citar las fuentes en un documento en el cual hay una parte escrita por ti, y entregarlo como trabajo propio.

Causas de ciberplagio académico

It8. Los profesores orientan demasiados trabajos en el curso.

It9. Algunos trabajos no aportan nada a mi formación.

It11. Generalmente dejo para última hora la realización de los trabajos académicos que me orientan los profesores.

It12. La aproximación de las fechas topes para entregar un trabajo, me llevan a plagiar un poco.

It13. He recibido asignaturas en la universidad o facultad para desarrollar conocimientos y habilidades para hacer uso de la información de forma ética y legal.

It14. Los profesores hacen un escaso seguimiento de los trabajos académicos orientados.

It15. Los profesores comprueban los trabajos académicos utilizando un software antiplagio, para detectar citas incorrectas o posible plagio.

It16. Sé que me puede pasar si un profesor descubre que he copiado un trabajo.

It19. A veces tengo la tentación de plagiar de Internet, pues los demás lo están haciendo.

Actitudes hacia el ciberplagio académico

It21. Copiar y pegar de Internet o descargar un trabajo para entregar, ya es un hecho común y no es repudiable entre el estudiantado.

It22. En tiempos de declive moral y ético, es importante debatir temas como el plagio y el ciberplagio entre el estudiantado.

It26. Todo lo que se publica en Internet se puede copiar y/o descargar, por lo que no cometo ninguna violación.

It27. El plagio empobrece el espíritu investigador.

Estrategias y fuentes de búsqueda documental

It28. Cuando tengo que buscar información para realizar un trabajo, consulto en primer lugar, Internet.

It29. Cuando tengo que buscar información para hacer un trabajo, generalmente, al primer lugar a donde voy es a Google.

It32. Sé cómo buscar información en Google Académico.

It39. Cuando tengo que buscar información para realizar un trabajo, consulto en primer lugar, los libros de texto.

It41. Cuando encuentro una página en Internet que me sirve para realizar un trabajo, dejo de buscar más información inmediatamente.

It46. Normalmente voy a la biblioteca a buscar información para realizar un trabajo.

Conocimientos y habilidades en el manejo de información

It34. Cuando tengo que hacer un trabajo voy a Internet, busco información, la copio y la pego en un documento y entrego el trabajo tal cual.

It36. Confío siempre en la veracidad de lo que encuentro en Internet.

It38. Conozco las normas establecidas para hacer un uso ético y legal de la información, por ejemplo (el Derecho de Autor y las Licencias Creative Commons). 
It40. Cuando realizo un trabajo consulto los estilos y normas de redacción de bibliografías (APA, ISO-690, o cualquier otro sistema) para elaborar correctamente las citas y referencias.

It47. Conozco el protocolo de las citas textuales y sus diferencias con las citas parafraseadas.

\section{Nota: Elaboración propia}

\section{Conclusiones}

La revisión de literatura permitió profundizar en la prevalencia, prácticas y causas del plagio académico en la era digital, tema de merecida atención por su extensión en los últimos años. Es un constructo complejo, multidimensional y basado en principios ético-morales que lo asocian a una época, una cultura y una sociedad determinada. Lo cual ha proporcionado una variedad de instrumentos para evaluar esta práctica, en su mayoría descriptivos y sin establecer una relación causa-efecto entre los factores. En este sentido, es el aporte más significativo del instumento concretado a partir de su adaptación y validación. Además de incluir aspectos relacionados con las habilidades informacionales como elemento básico para reducir la comisión de ciberplagio. Aunque su fiabilidad se obtuvo mediante datos aportados por estudiantes cubanos, puede ser adaptado o aplicado en otros contextos. También porque la validez de contenido fue avalada por expertos de diferentes países, aportándole una mirada más abarcadora. Se concluye que es un instrumento válido y fiable para analizar el ciberplagio en trabajos académicos uiversitarios, especialmente en el contexto cubano donde el tema ha sido poco explorado. Independientemente de que el análisis psicométrico arrojó resultados favorables, se recomienda para estudios futuros el Análisis Factorial Confirmatorio para esclarecer o corroborar el resultado del AFE.

\section{Referencias}

Awasthi, S. (2019). Plagiarism and Academic Misconduct: A Systematic Review. Journal of Library \& Information Technology, 39(2), 94-100. https://doi.org/10.1080/02602938.2014.966053

Belli, S., Raventós, C. L., \& Guarda, T. (2020). Plagiarism detection in the classroom: honesty and trust through the urkund and turnitin software. Advances in Intelligent Systems and Computing, 1137 AISC, 660-668. https://doi.org/10.1007/978-3-030-40690-5_63

Blasco, J. E., \& López, A. (2010). Validación mediante método Delphi de un cuestionario para conocer las experiencias e interés hacia las actividades acuáticas con especial atención al windsurf. (Número 12 (1)). https://bit.ly/3mJukiu

Boillos, M. (2020). Las caras del plagio inconsciente en la escritura académica [The faces of unconscious plagiarism in academic writing]. Educación XX1, 211-229. https://doi.org/10.5944/educXX1.25658

Brennan, T. (2015). The Effect of Turinitin . com on Non-Traditional , Graduate Student Awareness, Behavior and Trust by Terence Brennan. (Dissertation/Thesis). Faculty of the College of Education of Trident University International. Denver, Colorado.

Catro, A. M., Espiñeira, E. M., Lozada, L., \& Gerpe, E. M. (2019). El alumnado universitario ante políticas institucionales y de aula sobre plagio (Students from higher education institutions towards institutional and classroom) policies on plagiarism. Bordon. Revista de Pedagogia, 71(2), 139-153. https://doi.org/10.13042/Bordon.2019.69104

Cebrián-Robles, V., Raposo-Rivas, M., Cebrián-de-la-Serna, M., \& Sarmientos-Campos, J. A. (2018). Perception of academic plagiarism by Spanish University. Educación XX1, 21 (2), 105-129. https://doi.org/10.5944/educXX1.20062

Cebrián-Robles, V., Raposo-Rivas, M., \& Sarmiento-Campos, A. (2020). Study of the reasons for and measures to avoid plagiarism in young students. Revista de Curriculum y Formación del Profesorado, 24 (1). https://doi.org/10.30827/profesorado.v24i1.8572

Comas, R., \& Sureda, J. (2010). Academic Plagiarism : Explanatory Factors from Students ' Perspective. Journal of Academic Ethics, 8(3), 217-232. https://doi.org/10.1007/s10805-010-9121-0

Comas, R., \& Sureda, J. (2016). Prevalence and ability to recognize academic plagiarism among university students in economics. Profesional de la Información, 25, 616-622. https://doi.org/10.3145/epi.2016.jul.11

Comas, R., Sureda, J., Casero, A., \& Morey, M. (2011). La integridad académica entre el alumnado universitario español [Academic integrity among Spanish university students]. Estudios pedagógicos (Valdivia), 37, 207-225. https://doi.org/10.4067/S0718-07052011000100011

Cortés-Vera, J. J. (2019). Hacia universidades libres de plagio académico: un llamado a la acción participativa [Towards universities free of academic plagiarism: a call for participatory action] (G. Garciarena (ed.); Primera ed). http://www2.uacj.mx/publicaciones/\%0A

Espiñeira-Bellón, E. M., Muñoz-Cantero, J. M., Gerpe-Pérez, E. M., \& Castro-Pais, M. D. (2021). Ciberplagio como soporte digital en la realización de trabajos académicos (Cyber-plagiarism as digital support for the submission of academic writing). Grupo Comunicar, 29(68), 119-128. https://doi.org/10.3916/C68-2021-10

Fatima, A., Abbas, A., Ming, W., Hosseini, S., \& Zhu, D. (2019). Internal and External Factors of Plagiarism: Evidence 
from Chinese Public Sector Universities. Accountability in Research, 26(1), 1-16. https://doi.org/10.1080/08989621.2018.1552834

Fazilatfar, A., Elhambakhsh, S., \& Allami, H. (2018). An Investigation of the Effects of Citation Instruction to Avoid Plagiarism in EFL Academic Writing Assignments. Sage OpenOpen, 8(2). https://doi.org/10.1177/2158244018769958

González-Torres, P., García-García, M. A., \& Coque, J. (2018). El plagio entre el alumnado universitario: un caso exploratorio [Plagiarism among university students: an exploratory case]. En Libro de actas del XXVI Congreso Universitario de Innovación Educativa en Enseñanzas Técnicas (p. 106). https://bit.ly/32OM3fH

Hernández-Sampieri, R., Fernández-Collado, C., \& Baptista-Lucio, C. (2014). Metodología de la Investigación. En McGRAW- HILL Educación (Ed.), Journal of Chemical Information and Modeling (6ta ed., Vol. 53, Número 9). https://doi.org/10.1017/CBO9781107415324.004

Husain, F., Al-Shaibani, G., \& Mahfoodh, O. (2017). Perceptions of and Attitudes toward Plagiarism and Factors Contributing to Plagiarism: A Review of Studies. Journal of Academic Ethics. https://doi.org/10.1007/s10805-0179274-1

Keeney, S., Hasson, F., \& McKenna, H. (2006). Consulting the oracle: ten lessons from using the Delphi technique in nursing research. Journal of Advanced Nursing, 53(2), 205-212. https://doi.org/10.1111/j.1365-2648.2006.03716.x

Lacave, C., Molina, A., Fernández, M., \& Redondo, M. (2015). Análisis de la fiabilidad y validez de un cuestionario docente. Actas de las XXI Jornadas de la Enseñanza Universitaria de la Informática, 9, 136-143. https://bit.ly/31PIENF

Lloret-Segura, S., Ferreres-Traver, A., Hernández-Baeza, A., \& Tomás-Marco, I. (2014). El análisis factorial exploratorio de los ítems: Una guía práctica, revisada y actualizada. Anales de Psicologia, 30(3), 1151-1169. https://doi.org/10.6018/analesps.30.3.199361

López-Gómez, E. (2018). El método Delphi en la investigación actual en educación: una revisión teórica y metodológica. En Educación XX1: Vol. 21 (1) (pp. 17-40). https://doi.org/10.5944/educXX1.15536

Mengual-Andrés, S., Roig-Vila, R., \& Blasco, J. (2016). Delphi study for the design and validation of a questionnaire about digital competences in higher education. International Journal of Educational Technology in Higher Education. https://doi.org/10.1186/s41239-016-0009-y

Muñoz-Cantero, J. M., Rebollo-Quintela, N., Mosteiro-García, J., \& Ocampo-Gómez, C. I. (2019). Validación del cuestionario de atribuciones para la detección de coincidencias en trabajos académicos. RELIEVE - Revista Electrónica de Investigación y Evaluación Educativa, 25(1), 1-16. https://doi.org/10.7203/relieve.25.1.13599

Mut, B. (2011). La alfabetización informacional: una aproximación al ciberplagio académico entre el alumnado de bachillerato. Repositorio Institucional.

Olivia-Dumitrina, N., Casanovas, M., \& Capdevila, Y. (2019). Academic writing and the internet: Cyber-plagiarism amongst university students. Journal of New Approaches in Educational Research, 8(2), 112-125. https://doi.org/10.7821/naer.2019.7.407

Oran, N. T., Ozturk, H., Senol, S., \& Pelik, A. (2016). Academic dishonesty among health science school students. Nursing Ethics, 23(8), 919-931. https://doi.org/10.1177/0969733015583929

Prieto, G., \& Delgado, A. R. (2010). Fiabilidad y validez. Papeles del Psicologo, 31(1), 67-74. http://www.papelesdelpsicologo.es/pdf/1797.pdf

Pupovac, V., Bilic-zulle, L., Mavrinac, M., \& Petrovecki, M. (2010). Attitudes toward plagiarism among pharmacy and medical biochemistry students - cross-sectional survey study. Biochemia Medica, 20(3), 307-313.

Rogerson, A., \& McCarthy, G. (2017). Using Internet based paraphrasing tools: Original work, patchwriting or facilitated plagiarism? International Journal for Educational Integrity, 13(1). https://doi.org/10.1007/s40979-016-0013-y

Simeon, T. (2014). Academic Honesty in the Digital Age (Número May). (Dissertation/Thesis). Indiana University of Pennsylvania.

Singh, N. (2017). Level of awareness among veterinary students of GADVASU towards plagiarism: A case study. Electronic Library, 35(5), 899-915. https://doi.org/10.1108/EL-06-2016-0132

Sureda, J., \& Comas, R. (2007). Ciberplagio académico: la generación "copiar y pegar"[Academic cyberplagiarism: the copy and paste generation]. Actas digitales del III Congreso Online del Observatorio para la Cbersociedad: Conocimiento Abierto. Sociedad Libre.

Sureda, J., Comas, R., Sureda, J., \& Oliver, M. (2006). Ciberplagio Académico . Una aproximación al estado de los conocimientos [Academic Cyberplagiarism. An approach to the state of knowledge]. Revista TEXTOS de la CiberSociedad, 10. Temática Variada. https://www.researchgate.net/publication/28139235\%0ACiber-Plagio

Torres-Diaz, J. C., Duart, J. M., \& Hinojosa-Becerra, M. (2018). Plagiarism, Internet and Academic Success at the University. Journal of New Approaches in Educational Research, 7(2), 98-104. https://doi.org/10.7821/naer.2018.7.324 